\title{
Sinus Node Functional Assessment
}

National Cancer Institute

\section{Source}

National Cancer Institute. Sinus Node Functional Assessment. NCI Thesaurus. Code C100053.

Description of electrical activity and conduction involving the sinoatrial node. (ACC) 